\title{
Identification of genes and signaling pathways associated with squamous cell carcinoma by bioinformatics analysis
}

\author{
LI SHEN $^{1,2}$, LINBO LIU ${ }^{1}$, ZHENYONG YANG ${ }^{2}$ and NAN JIANG ${ }^{2}$ \\ ${ }^{1}$ Department of Plastic Surgery, The First Affiliated Hospital of Zhengzhou University; ${ }^{2}$ Department of Plastic and \\ Aesthetic Surgery, The Fifth Affiliated Hospital of Zhengzhou University, Zhengzhou, Henan 450052, P.R. China
}

Received December 18, 2014; Accepted November 2, 2015

DOI: 10.3892/ol.2015.4051

\begin{abstract}
The present study aimed to investigate the genes and signaling pathways associated with squamous cell carcinoma (SCC) by bioinformatics analysis. For this purpose, the GSE2503 was downloaded from the Gene Expression Omnibus database, and the differentially expressed genes (DEGs) between 6 normal skin and 5 SCC samples were analyzed using the Linear Models for Microarray Data package. Gene Ontology (GO) and pathway enrichment analysis of DEGs were performed, followed by functional annotation and construction of a protein-protein interaction (PPI) network. Subnetwork modules were subsequently identified and analyzed. A total of 181 DEGs, including 95 upregulated and 86 downregulated DEGs, were identified, in addition to $20 \mathrm{GO}$ biological processes terms enriched by upregulated DEGs and 14 enriched by downregulated DEGs. The upregulated DEGs were enriched in 18 pathways, and the downregulated DEGs were enriched in 7 pathways. Following functional annotation, three upregulated transcription factors (TFs), including hypoxia inducible factor 1, alpha subunit (HIF 1A), and six downregulated TFs were identified. In the PPI network and subnetwork, matrix metallopeptidase 1 (MMP1), also known as interstitial collagenase, and interleukin 8 (IL8) were the hub genes with the highest degree of connectivity (degree $=8$ ). Integrin alpha $(I T G A) 6$ and 2 were enriched in several pathways, including focal adhesion and extracellular matrix-receptor interaction. DEGs of SCC were primarily enriched in pathways associated with cancer and cell adhesion. Therefore, DEGs such as IL8, MMP1, HIF1A, ITGA6 and ITGA2 may be potential targets for the diagnosis and treatment of SCC.
\end{abstract}

Correspondence to: Dr Linbo Liu, Department of Plastic Surgery, The First Affiliated Hospital of Zhengzhou University, 1 Jianshe East Road, Zhengzhou, Henan 450052, P.R. China E-mail: linboliudr@163.com

Key words: squamous cell carcinoma, differentially expressed genes, pathway enrichment analysis, protein-protein interaction

\section{Introduction}

Squamous cell carcinoma (SCC) is a histologically distinct type of cancer (1). It arises from the uncontrolled proliferation of epithelial cells or cells exhibiting cytological or tissue architectural characteristics of SC differentiation, including the presence of keratin, tonofilament bundles or desmosomes, which are structures involved in cell-cell adhesion (1). SCC occurs in numerous tissues, including the lips, mouth, esophagus, lungs, urinary bladder and prostate (2). Of all the cases of SCC, $2.5 \%$ become metastatic and lead to substantial morbidity, which constitutes a considerable economic burden to the healthcare system (3). The incidence of SCC has notably increased worldwide over the last decade (4). Thus, an improved understanding of the underlying molecular mechanisms and gene networks involved in the development and progression of skin SCC is required.

Numerous studies on the mechanisms and therapeutic strategies for the treatment of SCC have been reported to date (5-8). Exposure to ultraviolet radiation, a potent mutagen and DNA-damaging agent, is considered to be a significant risk factor for the development of $\operatorname{SCC}(5,6)$. Carcinogenesis is a multistep process. During tumor progression, multiple genes experience up- or downregulation (7). A number of genes and signaling pathways involved in the progression of SCC have been previously identified (3,6-9). Streit et al (8) reported that thrombospondin-1 (TSP-1) was an effective inhibitor of angiogenesis and tumor growth in carcinomas of the skin. In addition, the authors observed that the expression of TSP-1 was downregulated in patients with SCC. Previous studies have established that the transforming growth factor $\beta$-mothers against decapentaplegic homolog 4 (SMAD4) signaling pathway is required for effective epithelial wound healing, and a conditional deletion of SMAD4 in the epidermis causes defects in skin wound healing, which are accompanied by spontaneous skin inflammation and SCC (6). Additionally, talin 1 and laminin alpha 3, which participate in signaling pathways associated with adhesion and migration, have been previously observed to be overexpressed in SCC (9). Padilla et al (3) and Nindl et al (7) selected differentially expressed genes (DEGs) between SCC and normal skin (NO) samples using the microarray expression profile dataset GSE2503. However, the interactions 
between the carcinogenic genes that lead to SCC remain to be elucidated.

In the present study, GSE2503 was downloaded and used to identify the DEGs between SCC and NO samples, in order to investigate the underlying molecular mechanisms of SCC. Subsequently, functional enrichment analysis and functional annotation were performed. In addition, protein-protein interaction (PPI) networks and subnetworks were constructed and analyzed, in order to study and identify target genes for the diagnosis and treatment of SCC. The results of the present study may facilitate the understanding of the mechanisms responsible for the carcinogenesis and development of SCC. Furthermore, the genes identified in the present study may serve as biomarkers for the diagnosis and prognosis of SCC.

\section{Materials and methods}

Affymetrix microarray data. The microarray expression profile dataset GSE2503 $(3,7)$, which is based on the Gene Expression Omnibus (GEO) Platform 96 GeneChip ${ }^{\circledR}$ Human Genome U133A 2.0 Array (Affymetrix, Inc., Santa Clara, CA, USA), was downloaded from the National Center of Biotechnology Information GEO database (http://www. ncbi.nlm.nih.gov/geo/). The dataset contained 15 samples, including six NO, four actinic keratosis and five SCC. In the present study, the SCC and NO samples were analyzed by bioinformatics methods.

Data preprocessing and differential expression analysis. The original array data were converted into expression measures. Background correction, quartile data normalization and probe summarization were performed using the Robust Multi-array Average (10) algorithm in the $\mathrm{R}$ affy package (https://www.bioconductor.org/packages/release/bioc/html/affy.html). Paired t-test based on the Linear Models for Microarray Data package (https://bioconductor.org/packages/release/bioc/html/limma.html) (11) in $\mathrm{R}$ (https://www.r-project.org/) was used to identify DEGs between SCC and NO samples. Multiple testing correction was performed with the Benjamini-Hochberg method (12) to obtain the adjusted P-value. Subsequently, $\log _{2}$-fold change $\left(\log _{2} \mathrm{FC}\right)$ was calculated. Only those genes exhibiting $\mid \log _{2} \mathrm{FCl}>1.0$ and adjusted $\mathrm{P}<0.05$ were regarded as DEGs.

Gene ontology and pathway enrichment analysis. Gene Ontology (GO; http://geneontology.org/) (13) is a tool for unification of biology that collects structured, defined and controlled vocabulary for large-scale gene annotation. The Kyoto Encyclopedia of Genes and Genomes (KEGG; http://www.genome.jp/kegg/) (14) knowledge database is a collection of online databases regarding genomes, enzymatic pathways and biological chemicals. The Database for Annotation, Visualization and Integrated Discovery (DAVID; https://david.ncifcrf.gov/) (15) contains a comprehensive set of functional annotation tools that have been developed for associating functional terms with lists of genes via clustering algorithms. In order to analyze the identified DEGs at the functional level, GO enrichment and KEGG pathway analysis were performed using the DAVID online tool. $\mathrm{P}<0.05$ was set as the threshold value.
Functional annotation of DEGs. Functional annotation analysis was performed to determine whether the identified DEGs functioned as transcription factors (TFs). The Tumor Suppressor Gene Database (TSGene; http://bioinfo. mc.vanderbilt.edu/TSGene/) (16) integrates TSGs with large-scale experimental evidence to provide a comprehensive resource for the investigation of TSGs and their implication in the molecular mechanisms of cancer. The Tumor Associated Gene (TAG; http://www.binfo.ncku.edu.tw/TAG/GeneDoc. php) database (17) contains information regarding genes involved in carcinogenesis. In the present study, all the oncogenes and TSGs known to date were extracted from the TAG and TSGene databases.

PPI network construction. The Search Tool for the Retrieval of Interacting Genes (STRING; http://string-db.org/) database (18) is a precomputed global resource designed to evaluate PPI information. In the present study, the STRING online tool was used to analyze the PPI of DEGs, and those experimentally validated interactions with a combined score $>0.4$ were selected as significant.

The majority of the PPI networks in the biological network constructed were observed to obey the scale-free attribution (19). Thus, the degree of connectivity was statistically analyzed in networks using cytoscape (www.cytoscape. org) (20), to obtain the significant nodes or hub proteins (21) in the PPI networks.

Subnetwork identification and functional enrichment analysis. The BioNet package (https://www.bioconductor. org/packages/release/bioc/html/BioNet.html) (22) provides a comprehensive set of methods for the integrated analysis of gene expression data and biological networks. The GeneAnswers (23) package (https://www.bioconductor. org/packages/release/bioc/html/GeneAnswers.html) facilitates the understanding of the associations between a list of genes and any relevant annotations.

In the present study, the BioNet package was used to identify the subnetworks in the constructed PPI networks, with a threshold false discovery rate $($ FDR $)<0.001$. Subsequently, the GeneAnswers package based on Entrez Gene ID (http://www.ncbi.nlm.nih.gov/gene) was used to identify over-represented GO terms with an FDR $<0.05$, and significantly enriched pathways with $\mathrm{P}<0.05$. Subsequently, data integration and network visualization were performed to obtain heat maps and association networks of the results derived from the enrichment analysis and the corresponding genes.

\section{Results}

Identification of 181 DEGs. Following data preprocessing, a total of 181 genes that were differentially expressed in SCC compared with NO were identified. These DEGs included 95 upregulated and 86 downregulated genes.

GO and pathway enrichment analysis. A total of $20 \mathrm{GO}$ biological processes (BPs) terms enriched by the upregulated DEGs (including cell adhesion) and 14 GO BPs terms enriched by the downregulated DEGs (including oxidation-reduction processes) 
Table I. GO functional and KEGG pathway enrichment analyses for the most significantly up- and downregulated DEGs.

\begin{tabular}{|c|c|c|c|c|}
\hline Category & Term & Description & $\begin{array}{c}\text { Degree of } \\
\text { connectivity }\end{array}$ & P-value \\
\hline \multicolumn{5}{|c|}{ Upregulated DEGs } \\
\hline $\mathrm{BP}$ & GO:0007155 & Cell adhesion & 18 & $1.53 \times 10^{-5}$ \\
\hline BP & GO:0006954 & Inflammatory response & 12 & $5.69 \times 10^{-5}$ \\
\hline BP & GO:0050900 & Leukocyte migration & 10 & $4.37 \times 10^{-6}$ \\
\hline BP & GO:0030216 & Keratinocyte differentiation & 7 & $3.74 \times 10^{-6}$ \\
\hline $\mathrm{BP}$ & GO:0032602 & Chemokine production & 5 & $2.27 \times 10^{-5}$ \\
\hline \multicolumn{5}{|c|}{ Downregulated DEGs } \\
\hline BP & GO:0055114 & Oxidation-reduction & 10 & $4.33 \times 10^{-4}$ \\
\hline BP & GO:0042391 & Regulation of membrane potential & 7 & $1.23 \times 10^{-3}$ \\
\hline BP & GO:0006898 & Receptor-mediated endocytosis & 4 & $5.09 \times 10^{-3}$ \\
\hline BP & GO:0006805 & Metabolism of xenobiotics & 4 & $9.58 \times 10^{-3}$ \\
\hline BP & GO:0003215 & Cardiac right ventricle morphogenesis & 3 & $7.73 \times 10^{-5}$ \\
\hline \multicolumn{5}{|c|}{ Upregulated DEGs } \\
\hline KEGG & 5200 & Signaling pathways in cancer & 8 & $9.37 \times 10^{-4}$ \\
\hline KEGG & 4810 & Regulation of actin cytoskeleton & 5 & $1.14 \times 10^{-2}$ \\
\hline KEGG & 4640 & Hematopoietic cell lineage & 4 & $2.36 \times 10^{-3}$ \\
\hline KEGG & 5219 & Bladder cancer & 3 & $2.41 \times 10^{-3}$ \\
\hline KEGG & 5412 & Arrhythmogenic right ventricular cardiomyopathy & 3 & $1.18 \times 10^{-2}$ \\
\hline \multicolumn{5}{|c|}{ Downregulated DGEs } \\
\hline KEGG & 480 & Metabolism of glutathione & 3 & $3.14 \times 10^{-3}$ \\
\hline KEGG & 330 & Metabolism of arginine and proline & 3 & $3.91 \times 10^{-3}$ \\
\hline KEGG & 980 & Metabolism of xenobiotics by cytochrome P450 & 3 & $8.41 \times 10^{-3}$ \\
\hline KEGG & 982 & Metabolism of drugs by cytochrome P450 & 3 & $9.08 \times 10^{-3}$ \\
\hline KEGG & 310 & Lysine degradation & 2 & $2.79 \times 10^{-2}$ \\
\hline
\end{tabular}

GO, Gene Ontology; KEGG, Kyoto Encyclopedia of Genes and Genomes; DEGs, differentially expressed genes; BP, biological process.

were identified by GO and pathway enrichment analysis. The most significant GO BPs terms are presented in Table I.

Table I also contains the most significantly enriched pathways of the upregulated and downregulated DEGs revealed by KEGG analysis. The upregulated DEGs were observed to be enriched in 18 pathways, while the downregulated DEGs were enriched in 7 pathways.

Functional annotation of DEGs. Upon analyzing the differential expression pattern of TFs and TAGs in SCC and NO samples, the present study identified a number of TFs, including hypoxia inducible factor 1, alpha subunit (HIF1A), aryl hydrocarbon receptor nuclear translocator-like 2 and paired-like homeodomain 1 , which were significantly upregulated, in addition to six TFs, including nuclear receptor subfamily 3 , hepatic leukemia factor and zinc finger protein 83, which were significantly downregulated in SCC. Among the upregulated DEGs, 11 genes were identified as TAGs. Of these, four were oncogenes and five were TSGs. The function of the remaining two genes identified in the analysis remains to be elucidated. Among the downregulated DEGs, five genes were identified as TSGs, including low-density lipoprotein receptor-related protein $1 \mathrm{~B}$ and proline dehydrogenase (oxidase) 1.
PPI network construction. Based on the information contained in the STRING database, 104 protein pairs were identified (Fig. 1). A total of 14 nodes were selected as hub proteins (degree $\geq 5$ ) in the PPI network, including matrix metallopeptidase 1 (MMP1) (also known as interstitial collagenase), keratin 6A (KRT6A) and interleukin 8 (IL8), which presented a degree of connectivity of 8 (Table II).

Subnetwork identification and functional enrichment analysis. As represented in Fig. 2, 43 nodes, 75 protein pairs and 9 hub proteins with a degree $\geq 6$ were identified in the subnetwork. The hub proteins, including IL8, MMP1 and KRT6A, are summarized in Table II.

Using heat maps (Figs. 3 and 4), the association between DEGs and BPs/signaling pathways was evaluated. For example, integrin alpha (ITGA) 6 and 2 were observed to be enriched in several BPs terms, including extracellular matrix (ECM)-receptor interaction and regulation of the KEGG signaling pathways of cell and focal adhesion.

\section{Discussion}

SCC is characterized by a high rate of proliferation and nodal metastasis (24). Therefore, early detection or prevention of 


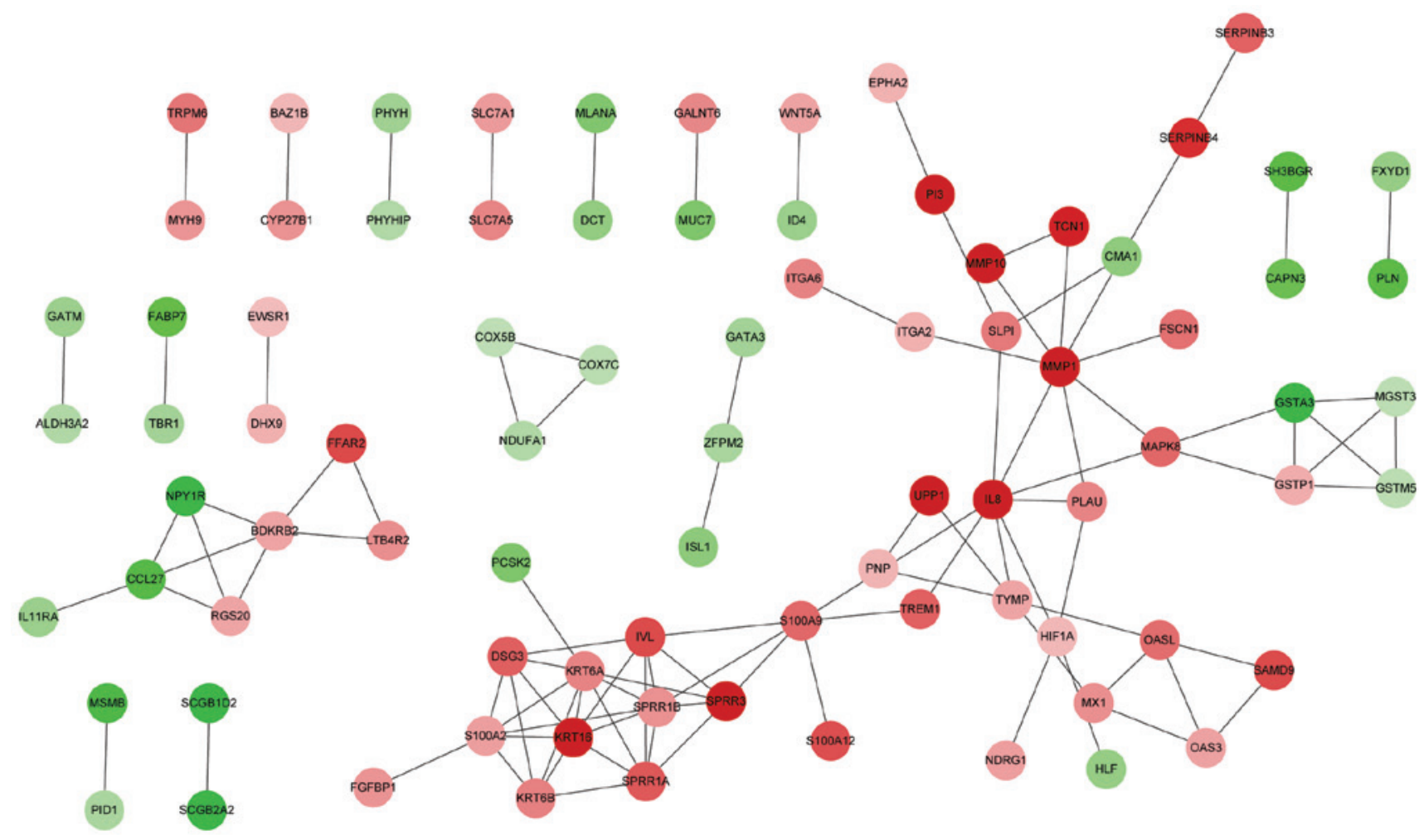

Figure 1. Constructed protein-protein interaction network of DEGs. Red, upregulated DEGs. Green, downregulated DEGs. DEGs, differentially expressed genes; ALDH3A2, aldehyde dehydrogenase 3 family, member A2; BAZ1B, bromodomain adjacent to zinc finger domain, 1B; BDKRB2, bradykinin receptor B2; CAPN3, calpain 3; CCL27, chemokine (C-C motif) ligand 27; CMA1, chymase 1, mast cell; COX5B, cytochrome $c$ oxidase subunit 5B; COX7C, COX 7C; CYP27B1, cytochrome P450, family 27, subfamily B, polypeptide 1; DCT, dopachrome tautomerase; DSG3, desmoglein 3; DHX9, DEAH (Asp-Glu-Ala-His) box helicase 9; EPHA2, ephrin type-A receptor 2; EWSR1, Ewing sarcoma RNA-binding protein 1; FABP7, fatty acid binding protein 7, brain; FFAR2, free fatty acid receptor 2; FGFBP1, fibroblast growth factor binding protein 1; FSCN1, fascin actin-bundling protein 1; FXYD1, FXYD domain containing ion transport regulator 1; GALNT6, polypeptide N-acetylgalactosaminyltransferase 6; GATA3, GATA binding protein 3; GATM, glycine amidinotransferase (L-arginine:glycine amidinotransferase); GSTA3, glutathione S-transferase alpha 3; GSTM5, GST Mu 5; GSTP1, GST Pi 1; HIF1A, hypoxia-inducible factor 1, alpha subunit; HLF, hepatic leukemia factor; ID4, inhibitor of DNA binding 4, dominant negative helix-loop-helix protein; IL8, interleukin 8; IL11RA, IL 11 receptor, alpha; ISL1, ISL LIM homeobox 1; ITGA2, integrin alpha 2; ITGA6, ITGA 6; IVL, involucrin; KRT6A, keratin 6A; KRT6B, KRT 6B; KRT16, KRT 16; LTB4R2, leukotriene B4 receptor 2; MAPK8, mitogen-activated protein kinase 8; MGST3, microsomal GST 3; MLANA, melan-A; MMP1, matrix metallopeptidase 1; MMP10, MMP 10; MSMB, microseminoprotein, beta-; MUC7, mucin 7, secreted; MX1, Mx dynamin-like guanosine triphosphate hydrolase 1; MYH9, myosin, heavy chain 9, non-muscle; NDRG1, N-myc downstream regulated 1; NDUFA1, nicotinamide adenine dinucleotide hydrogen dehydrogenase (ubiquinone) 1 alpha subcomplex; NPY1R, neuropeptide Y receptor Y1; OAS3, 2'-5'-oligoadenylate synthetase 3; OASL, OAS-like; PI3, peptidase inhibitor 3, skin derived; PID1, phosphotyrosine interaction domain containing 1; PLAU, plasminogen activator, urokinase; PLN, phospholamban; PNP, purine nucleoside phosphorylase; PHYH, phytanoyl-CoA 2-hydroxylase; PHYHIP, PHYH interacting protein; RGS20, regulator of G protein signaling 20; S100A2, S100 calcium-binding protein A2; S100A9, S100A 9; S100A12, S100A 12; SAMD9, sterile alpha motif domain containing 9; SCGB1D2, secretoglobin, family 1D, member 2; SCGB2A2, SCGB, family 2A, member 2; SERPINB3, serpin peptidase inhibitor, clade B (ovalbumin), member 3; SERPINB4, SERPINB 4; SH3BGR, SH3 domain binding glutamate-rich protein; SLC7A1, solute carrier family 7 (cationic amino acid transporter, Y+ system), member 1; SLC7A5, SLC7A 5; SLPI, secretory leukocyte protease inhibitor; SPRR1A, small proline-rich protein 1A; SPRR1B, SPRR 1B; SPRR3, SPRR 3; TBR1, T-box, brain, 1; TCN1, transcobalamin I (vitamin B12 binding protein, R binder family); TREM1, triggering receptor expressed on myeloid cells 1; TRPM6, transient receptor potential cation channel, subfamily M, member 6; TYMP, thymidine phosphorylase; UPP1, uridine phosphorylase 1; WNT5A, wingless-type mouse mammary tumour virus integration site family, member 5A; ZFPM2, zinc finger protein, FOG family member 2.

this disease may be the most effective approach to improve patients' prognosis. In the present study, a total of 181 DEGs that were differently expressed in SCC vs. NO samples were identified via the gene expression profile contained in GSE2503. The upregulated DEGs were enriched in BPs terms associated with cell adhesion and cancer signaling pathways. In the constructed PPI network and subnetwork, the hub genes $M M P 1$ and IL8 presented the highest degree of connectivity. Additionally, ITGA6 and ITGA2 were enriched in several pathways and GO BPs terms in the subnetworks. These results suggested that the above genes and signaling pathways may participate in the progression of SCC.

Cell adhesion is a common event in BPs (23). Alterations in the expression levels of cell-cell adhesion molecules have been previously proposed to contribute to the progression of malignant tumors (25). In the present study, IL8, one of the hub genes with the highest degree of connectivity, was observed to be enriched in the BP of cell adhesion. IL8 is a proinflammatory cytokine that promotes chemotaxis and degranulation in neutrophils (26). Overexpression of IL8 or its receptors has been previously observed in cancer cells, endothelial cells and tumor-associated macrophages, suggesting a regulatory function for IL8 within the tumor microenvironment (26). Notably, IL8 has been previously reported to be able to stimulate cellular proliferation and angiogenesis in head and neck SCC by acting in an autocrine or paracrine manner (27). Additionally, a previous study on cultured oropharyngeal SCC lines has suggested that the overexpression of IL8 may enhance 
Table II. Statistical analysis of the degrees of connectivity corresponding to the most significant hub genes identified in the protein-protein interaction network and subnetwork.

\begin{tabular}{lcc}
\hline Gene & Degree of connectivity & Adjusted P-value \\
\hline MMP1 & 8 & 0.012 \\
KRT6A & 8 & 0.028 \\
IL8 & 8 & 0.005 \\
SPRRIB & 7 & 0.031 \\
KRT16 & 7 & 0.001 \\
SPRR1A & 6 & 0.012 \\
IVL & 6 & 0.015 \\
S100A9 & 6 & 0.028 \\
S100A2 & 6 & 0.014 \\
\hline
\end{tabular}

IL8, interleukin 8; IVL, involucrin; KRT6A, keratin 6A; KRT16, KRT 16; MMP1, matrix metallopeptidase 1; S100A2, S100 calcium-binding protein A2; S100A9, S100A 9; SPRR1A, small proline-rich protein 1A; SPRR1B, SPRR B.

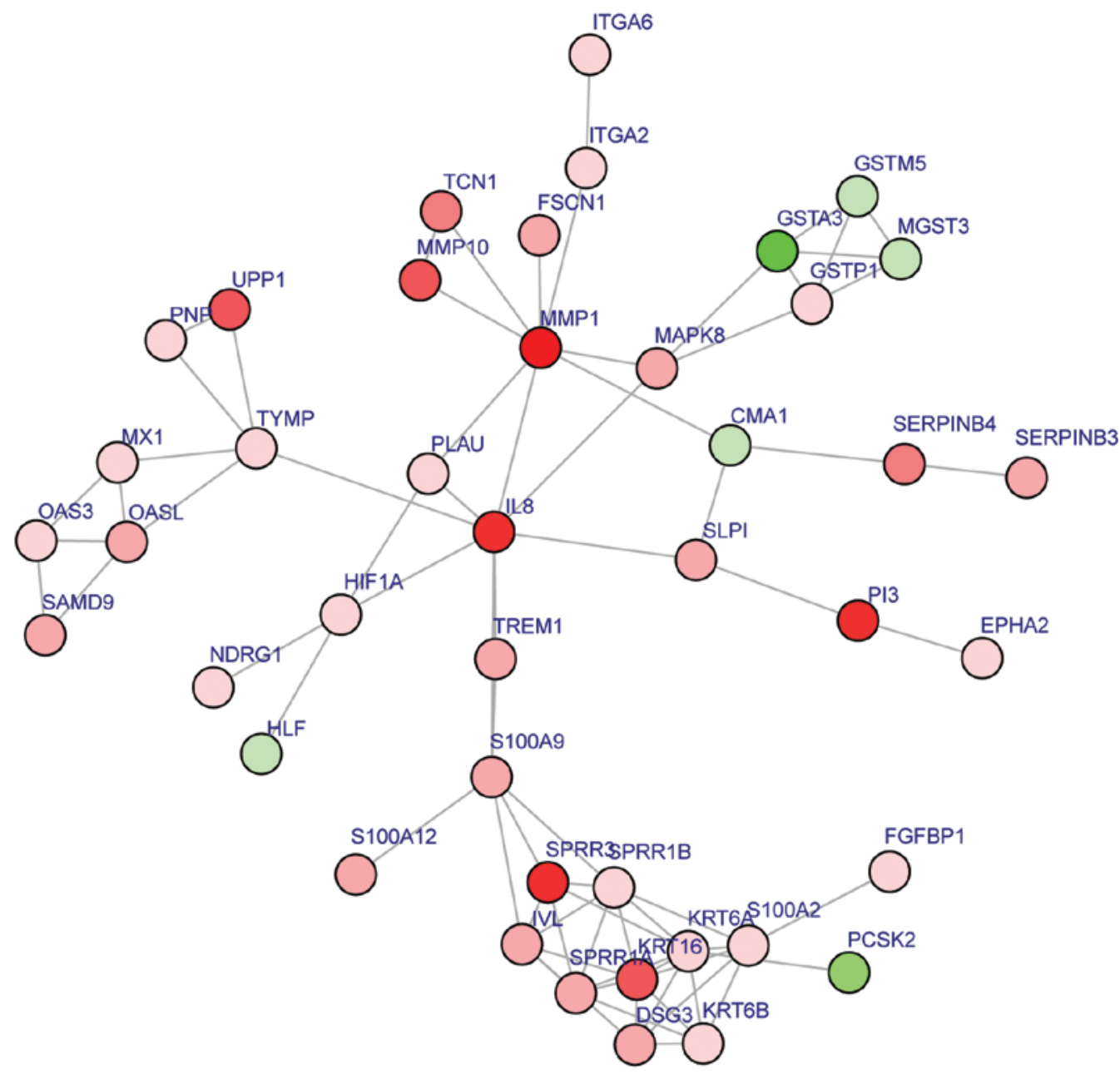

Figure 2. Constructed subnetwork of DEGs. Red, upregulated DEGs. Green, downregulated DEGs. DEGs, differentially expressed genes; CMA1, chymase 1, mast cell; DSG3, desmoglein 3; EPHA2, ephrin type-A receptor 2; FGFBP1, fibroblast growth factor binding protein 1; FSCN1, fascin actin-bundling protein 1; GSTA3, glutathione S-transferase alpha 3; GSTM5, GST Mu 5; GSTP1, GST Pi 1; H1F1A, hypoxia-inducible factor 1, alpha subunit; HLF, hepatic leukemia factor; IL8, interleukin 8; ITGA2, integrin alpha 2; ITGA6, ITG 6; IVL, involucrin; KRT6A, keratin 6A; KRT6B, KRT 6B; KRT16, KRT 16; MAPK8, mitogen-activated protein kinase 8; MGST3, microsomal GST 3; MMP1, matrix metallopeptidase 1; MMP10, MMP 10; MX1, Mx dynamin-like guanosine triphosphate hydrolase 1; NDRG1, N-myc downstream regulated 1; PCSK2, proprotein convertase subtilisin/kexin type 2; PI3, peptidase inhibitor 3, skin derived; OAS3, 2'-5'-oligoadenylate synthetase 3; OASL, OAS-like; PLAU, plasminogen activator, urokinase; PNP, purine nucleoside phosphorylase; S100A2, S100 calcium-binding protein A2; S100A9, S100A 9; S100A12, S100A 12; SAMD9, sterile alpha motif domain containing 9; SERPINB3, serpin peptidase inhibitor, clade B (ovalbumin), member 3; SERPINB4, SERPIN 4; SLPI, secretory leukocyte protease inhibitor; SPRR1A, small proline-rich protein 1A; SPRR1B, SPRR 1B; SPRR3, SPRR 3; TCN1, transcobalamin I (vitamin B12 binding protein, R binder family); TREM1, triggering receptor expressed on myeloid cells 1; TYMP, thymidine phosphorylase; UPP1, uridine phosphorylase 1. 


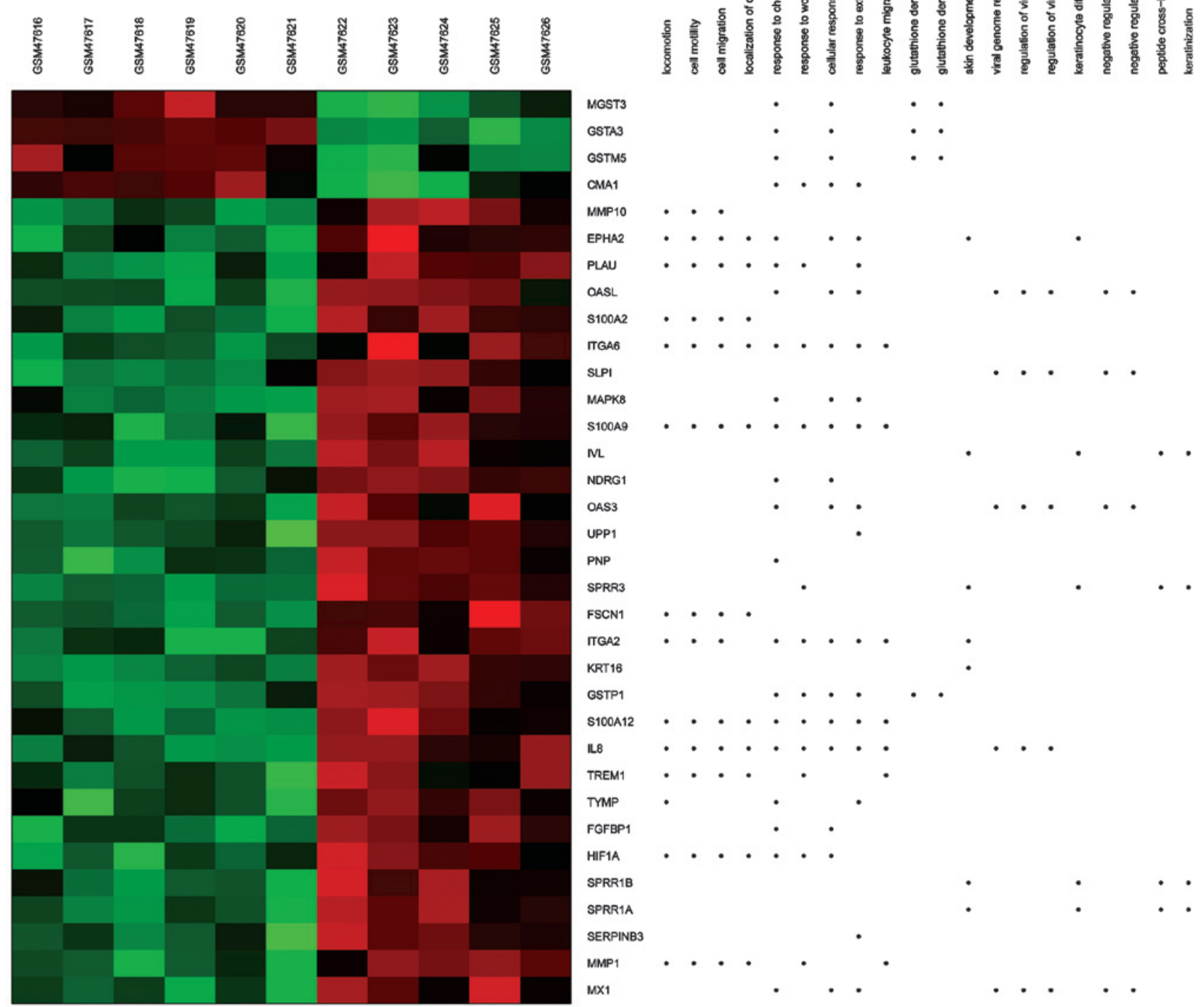

Figure 3. Heat map of Gene Ontology BPs generated via GeneAnswers. The dots indicate the BPs enriched by DEGs. Red, upregulated DEGs. Green, downregulated DEGs. BPs, biological processes; DEGs, differentially expressed genes; GSM, genome-scale model; CMA1, chymase 1, mast cell; EPHA2, ephrin type-A receptor 2; FGFBP1, fibroblast growth factor binding protein 1; FSCN1, fascin actin-bundling protein 1; GSTA3, glutathione S-transferase alpha 3; GSTM5, GST Mu 5; GSTP1, GST Pi 1; HIF1A, hypoxia-inducible factor 1, alpha subunit; IL8, interleukin 8; ITGA2, integrin alpha 2; ITGA6, ITGA 6; IVL, involucrin; KRT16, keratin 16; MAPK8, mitogen-activated protein kinase 8; MGST3, microsomal GST 3; MMP1, matrix metallopeptidase 1; MMP10, MMP 10; MX1, Mx dynamin-like guanosine triphosphate hydrolase 1; NDRG1, N-myc downstream regulated 1; PLAU, plasminogen activator, urokinase; PNP, purine nucleoside phosphorylase; OAS3, 2'-5'-oligoadenylate synthetase 3; OASL, OAS-like; S100A2, S100 calcium-binding protein A2; S100A9, S100A 9; S100A12, S100A 12; SERPINB3, serpin peptidase inhibitor, clade B (ovalbumin), member 3; SLPI, secretory leukocyte protease inhibitor; SPRR1A, small proline-rich protein 1A; SPRR3, SPRR 3; TREM1, triggering receptor expressed on myeloid cells 1; TYMP, thymidine phosphorylase; UPP1, uridine phosphorylase 1.

the pathogenicity of oropharyngeal SCC by promoting cell growth (28). Therefore, IL8 and the signaling pathways associated with cell adhesion appear to be closely connected with SCC, and they may be used as potential targets for the treatment of SCC.

Using the PPI networks and subnetworks constructed in the present study, MMPl was identified as one of the hub genes exhibiting the highest degree of connectivity. Additionally, $M M P 1$ was observed to be enriched in signaling pathways associated with cancer. MMP1 belongs to the MMP family, and participates in a variety of BPs, including cell proliferation, differentiation, migration, apoptosis and host defense (29). MMP1 has been previously associated with cancer invasion and metastasis, since it degrades fibrillar collagens, thus enabling the tumor to traverse the extracellular space (29). Notably, MMP1 is frequently detected in various types of cancer, and may be associated with advanced stages of the disease (30). For example, MMP1 appears to be overexpressed 

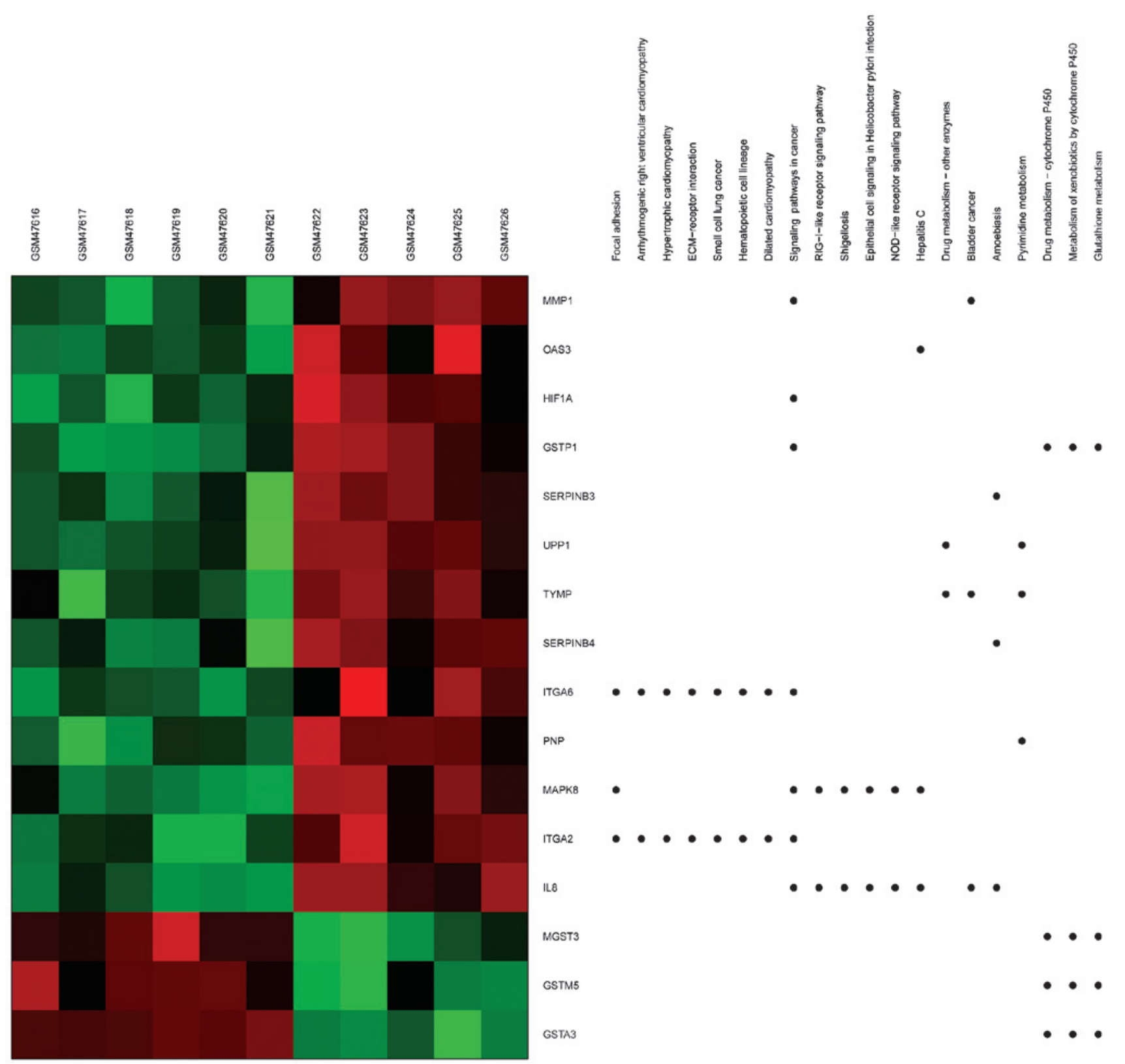

Figure 4. Heat map of Kyoto Encyclopedia of Genes and Genomes generated via GeneAnswers. The dots indicate the signaling pathways enriched by DEGs. Red, upregulated DEGs. Green, downregulated DEGs. DEGs, differentially expressed genes; ECM, extracellular matrix; GSM, genome-scale model; GSTA3, glutathione S-transferase alpha 3; GSTM5, GST Mu 5; GSTP1, GST Pi 1; HIF1A, hypoxia-inducible factor 1, alpha subunit; IL8, interleukin 8; ITGA2, integrin alpha 2; ITGA6, ITGA 6; MAPK8, mitogen-activated protein kinase 8; MGST3, microsomal GST 3; MMP1, matrix metallopeptidase 1; NOD, nucleotide-binding oligomerization; OAS3, 2'-5'-oligoadenylate synthetase 3; PNP, purine nucleoside phosphorylase; RIG-I, retinoic acid-inducible gene 1; SERPINB3, serpin peptidase inhibitor, clade B (ovalbumin), member 3; SERPINB4, SERPINB 4; TYMP, thymidine phosphorylase; UPP1, uridine phosphorylase 1.

in skin cancer, according to the studies by Nindl et al (7). Taken together, these data support the hypothesis that MMP1 is a candidate molecular marker associated with SCC.

In the present study, the TF HIFlA was identified to be overexpressed in SCC. HIFIA functions as a TF in response to cellular hypoxia, and participates in BPs associated with tumor angiogenesis and pathophysiology of ischemic disease (31). It has been previously reported that HIFlA may be a predictor of disease progression in esophageal SCC (32). Fillies et al (33) suggested that the overexpression of HIF1A may be an indicator of favorable prognosis in SCC of the oral cavity. Accordingly, HIFlA may be an important TF associated with SCC.
The present study also revealed that ITGA6 and ITGA2 were enriched in several GO BPs terms, including regulation of cell adhesion and migration, and KEGG signaling pathways of focal adhesion and ECM-receptor interaction. The protein product of the ITGA6 gene is the integrin alpha chain alpha 6 (34). Integrins have a significant role in cell adhesion and migration (34), and different combinations of integrins act as receptors of certain ECM proteins (35). Integrins participate in a number of BPs (including cell adhesion, cell migration, blood clotting and tissue organization) and cancer processes (including cell migration, metastasis and invasion) (34). ITGA6 interacts with the ECM protein laminin, and is involved in the regulation of cell adhesion, growth 
and migration (36). The role of ITGA6 in cancer development has been widely documented. Friedrichs et al (37) observed that the overexpression of ITGA6 was associated with unfavorable prognosis in patients with breast cancer. In addition, previous studies have reported that ITGA6 is highly expressed in esophageal SCC tissues and participates in the tumorigenesis of esophageal SCC (38). Therefore, ITGA6 may be a potential target gene for the treatment of SCC. Notably, ITGA2 encodes a cell adhesion molecule termed $\alpha 2 \beta 1$ integrin receptor, which enables the interaction of the cells with the ECM and mediates the signaling events occurring within the ECM (39). Recent studies have indicated that the ITGA2 gene is associated with various types of cancer, including colorectal (40) and breast cancer (41). In addition, Beaulieu (42) reported that ITGA2 was expressed in colon cancer cell lines, and participated in the proliferation and migration of these cells. There are limited studies on the effects of ITGA2 on SCC thus far (42). However, it may be speculated that ITGA2 may be a key gene, along with ITGA6, in the progression of SCC.

In conclusion, the results of the present study provide a comprehensive bioinformatics analysis of DEGs that may be involved in SCC. The results of the current study may contribute to understand the underlying molecular mechanisms that lead to SCC. Furthermore, the DEGs identified in the present study, including IL8, MMPI, HIFIA, ITGA6 and $I T G A 2$, and certain signaling pathways associated with focal adhesion and ECM-receptor interaction, may be potential targets for the diagnosis and treatment of SCC.

However, the present study has a number of limitations. Thus, the size of the sample employed in the microarray analysis was small, which may generate a high number of false positive results. Additionally, the present study lacked experimental verification. Therefore, further genetic and experimental studies with larger sample sizes are required to confirm the findings of the present study.

\section{Acknowledgements}

The authors would like to thank FengHe (ShangHai) Information Technology Co., Ltd. (Shanghai, China) for their advice and support.

\section{References}

1. Rudolph R and Zelac DE: Squamous cell carcinoma of the skin. Plast Reconstr Surg 114: 82e-94e, 2004.

2. Joshi SK, Bhadauria RS, Jadon G and Diwaker AK: Introduction to neoplasm: 'Tumor classificatio'\% A review article. IJARPB 2: 227-263, 2012.

3. Padilla RS, Sebastian S, Jiang Z, Nindl I and Larson R: Gene expression patterns of normal human skin, actinic keratosis, and squamous cell carcinoma: A spectrum of disease progression. Arch Dermatol 146: 288-293, 2010.

4. Diepgen TL and Mahler V: The epidemiology of skin cancer. Br J Dermatol 146 (Suppl 61): S1-S6, 2002.

5. Armstrong BK and Kricker A: The epidemiology of UV induced skin cancer. J Photochem Photobiol B 63: 8-18, 2001.

6. Owens P, Engelking E, Han G, Haeger SM and Wang XJ: Epidermal Smad4 deletion results in aberrant wound healing. Am J Pathol 176: 122-133, 2010.

7. Nindl I, Dang C, Forschner T, Kuban RJ, Meyer T, Sterry W and Stockfleth E: Identification of differentially expressed genes in cutaneous squamous cell carcinoma by microarray expression profiling. Mol Cancer 5: 30, 2006.
8. Streit M, Velasco P, Brown LF, Skobe M, Richard L, Riccardi L, Lawler J and Detmar M: Overexpression of thrombospondin-1 decreases angiogenesis and inhibits the growth of human cutaneous squamous cell carcinomas. Am J Pathol 155: 441-452, 1999.

9. Snijders AM, Schmidt BL, Fridlyand J, Dekker N, Pinkel D, Jordan RC and Albertson DG: Rare amplicons implicate frequent deregulation of cell fate specification pathways in oral squamous cell carcinoma. Oncogene 24: 4232-4242, 2005.

10. Irizarry RA, Hobbs B, Collin F, Beazer-Barclay YD, Antonellis KJ, Scherf U and Speed TP: Exploration, normalization, and summaries of high density oligonucleotide array probe level data. Biostatistics 4: 249-264, 2003.

11. Diboun I, Wernisch L, Orengo CA and Koltzenburg M: Microarray analysis after RNA amplification can detect pronounced differences in gene expression using limma. BMC Genomics 7: 252, 2006.

12. Benjamini Y and Hochberg Y: Controlling the false discovery rate: A practical and powerful approach to multiple testing. J R Stat Soc Series B Stat Methodol 57: 289-300, 1995.

13. Ashburner M, Ball CA, Blake JA, Botstein D, Butler $\mathrm{H}$, Cherry JM, Davis AP, Dolinski K, Dwight SS, Eppig JT, et al; The gene ontology consortium: Gene ontology: Tool for the unification of biology. Nat Genet 25: 25-29, 2000.

14. Kanehisa M and Goto S: KEGG: Kyoto encyclopedia of genes and genomes. Nucleic Acids Res 28: 27-30, 2000.

15. Huang DW, Sherman BT, Tan Q, Collins JR, Alvord WG, Roayaei J, Stephens R, Baseler MW, Lane HC and Lempicki RA: The DAVID Gene Functional Classification Tool: A novel biological module-centric algorithm to functionally analyze large gene lists. Genome Biol 8: R183, 2007.

16. Zhao M, Sun J and Zhao Z: TSGene: A web resource for tumor suppressor genes. Nucleic Acids Res 41 (D1): D970-D976, 2013.

17. Chen JS, Hung WS, Chan HH, Tsai SJ and Sun HS: In silico identification of oncogenic potential of fyn-related kinase in hepatocellular carcinoma. Bioinformatics 29: 420-427, 2013.

18. von Mering C, Huynen M, Jaeggi D, Schmidt S, Bork P and Snel B: STRING: A database of predicted functional associations between proteins. Nucleic Acids Res 31: 258-261, 2003.

19. Yook SH, Oltvai ZN and Barabási AL: Functional and topological characterization of protein interaction networks. Proteomics 4: 928-942, 2004.

20. Shannon P, Markiel A, Ozier O, Baliga NS, Wang JT, Ramage D, Amin N, Schwikowski B and Ideker T: Cytoscape: A software environment for integrated models of biomolecular interaction networks. Genome Res 13: 2498-2504, 2003.

21. He $X$ and Zhang $\mathrm{J}$ : Why do hubs tend to be essential in protein networks? PLoS Genet 2: e88, 2006.

22. Beisser D, Klau GW, Dandekar T, Müller T and Dittrich MT: BioNet: An R-Package for the functional analysis of biological networks. Bioinformatics 26: 1129-1130, 2010.

23. Feng G, Du P, Krett NL, Tessel M, Rosen S, Kibbe WA and Lin SM: A collection of bioconductor methods to visualize gene-list annotations. BMC Res Notes 3: 10, 2010.

24. Wong TS, Liu XB, Wong BY, Ng RW, Yuen AP and Wei WI: Mature miR-184 as potential oncogenic microRNA of squamous cell carcinoma of tongue. Clin Cancer Res 14: 2588-2592, 2008.

25. Birchmeier W, Weidner KM, Hülsken J and Behrens J: Molecular mechanisms leading to cell junction (cadherin) deficiency in invasive carcinomas. Semin Cancer Biol 4: 231-239, 1993.

26. Waugh DJ and Wilson C: The interleukin-8 pathway in cancer. Clin Cancer Res 14: 6735-6741, 2008.

27. Richards BL, Eisma RJ, Spiro JD, Lindquist RL and Kreutzer DL: Coexpression of interleukin-8 receptors in head and neck squamous cell carcinoma. Am J Surg 174: 507-512, 1997.

28. Smith DR, Polverini PJ, Kunkel SL, Orringer MB, Whyte RI, Burdick MD, Wilke CA and Strieter RM: Inhibition of interleukin 8 attenuates angiogenesis in bronchogenic carcinoma. J Exp Med 179: 1409-1415, 1994.

29. Kurahara S, Shinohara M, Ikebe T, Nakamura S, Beppu M, Hiraki A, Takeuchi H and Shirasuna K: Expression of MMPS, MT-MMP, and TIMPs in squamous cell carcinoma of the oral cavity: Correlations with tumor invasion and metastasis. Head Neck 21: 627-638, 1999.

30. Tsuchiya N, Narita S, Kumazawa T, Inoue T, Ma Z, Tsuruta H, Saito M, Horikawa Y, Yuasa T, Satoh S, et al: Clinical significance of a single nucleotide polymorphism and allelic imbalance of matrix metalloproteinase-1 promoter region in prostate cancer. Oncol Rep 22: 493-499, 2009. 
31. Semenza GL: HIF-1: Mediator of physiological and pathophysiological responses to hypoxia. J Appl Physiol (1985) 88: 1474-1480, 2000.

32. Yu ZT,Zhao HF and Shang XB: Expression of hypoxia-inducible factor-1alpha and vessel endothelial grow th factor in esophageal squamous cell carcinoma and clinico-pathological significance thereof. Zhonghua Yi Xue Za Zhi 88: 2465-2469, 2008 (In Chinese).

33. Fillies T, Werkmeister R, van Diest PJ, Brandt B, Joos U and Buerger H: HIF1-alpha overexpression indicates a good prognosis in early stage squamous cell carcinomas of the oral floor. BMC Cancer 5: 84, 2005.

34. Pawar SC, Demetriou MC, Nagle RB, Bowden GT and Cress AE: Integrin alpha6 cleavage: A novel modification to modulate cel migration. Exp Cell Res 313: 1080-1089, 2007.

35. Miranti $\mathrm{CK}$ and Brugge JS: Sensing the environment: $A$ historical perspective on integrin signal transduction. Nat Cell Biol 4: E83-E90, 2002.

36. Jauliac S, López-Rodriguez C, Shaw LM, Brown LF, Rao A and Toker A: The role of NFAT transcription factors in integrin-mediated carcinoma invasion. Nat Cell Biol 4: 540-544, 2002.
37. Friedrichs K, Ruiz P, Franke F, Gille I, Terpe HJ and Imhof BA: High expression level of alpha 6 integrin in human breast carcinoma is correlated with reduced survival. Cancer Res 55: 901-906, 1995.

38. Kwon J, Lee TS, Lee HW, Kang MC, Yoon HJ, Kim JH and Park JH: Integrin alpha 6: A novel therapeutic target in esophageal squamous cell carcinoma. Int J Oncol 43: 1523-1530, 2013.

39. Gürkan A, Emingil G, Afacan B, Berdeli A and Atilla G: Alpha 2 integrin gene (ITGA2) polymorphism in renal transplant recipients with and without drug induced gingival overgrowth. Arch oral biol 59: 283-288, 2014

40. Gerger A, Hofmann G, Langsenlehner U, Renner W, Weitzer W, Wehrschütz M, Wascher T, Samonigg H and Krippl P: Integrin alpha-2 and beta-3 gene polymorphisms and colorectal cancer risk. Int J Colorectal Dis 24: 159-163, 2009.

41. Langsenlehner U, Renner W, Yazdani-Biuki B, Eder T, Wascher TC, Paulweber B, Clar H, Hofmann G, Samonigg H and Krippl P: Integrin alpha-2 and beta-3 gene polymorphisms and breast cancer risk. Breast Cancer Res Treat 97: 67-72, 2006

42. Beaulieu JF: Integrins and human intestinal cell functions. Front Biosci 4: D310-D321, 1999. 